\title{
Organoaxial partial rotation of duodenum with midgut malrotation in an adult
}

\author{
Luckshika Udeshani Amarakoon ${ }^{1}$, MBBs, Baj Gamage Anushka Rathnamali ${ }^{1}$, MBBS, \\ Jasin Arachchige Saman Bingumal Jayasundara ${ }^{1}$, MD, MRCs, Ajith de Silva ${ }^{1}$, MS, FRCS
}

\begin{abstract}
Midgut malrotation includes a range of developmental abnormalities that occur during fetal intestinal rotation. Manifestations of intestinal malrotation are generally seen in the paediatric population and are uncommon in adults. Symptomatic patients may present with either acute abdominal pain due to midgut volvulus, or chronic abdominal pain due to proximal midgut partial obstruction in the presence of congenital bands. A limited number of paediatric cases of duodenal occlusion due to duodenal malrotation has been previously reported in the medical literature. We herein report the case of a 57-year-old woman who presented with duodenal obstruction due to organoaxial partial rotation of the distal duodenum associated with midgut malrotation. This is probably the first of such a case diagnosed in adulthood reported in the medical literature. Our patient underwent Roux-en-Y duodenojejunostomy and had symptomatic relief following the successful surgery.
\end{abstract}

Keywords: duodenal obstruction, midgut malrotation

\section{INTRODUCTION}

Midgut malrotation occurs as a range of embryological developmental abnormalities that happen during fetal intestinal rotation. The majority of symptomatic patients present during the first year of life. ${ }^{(1)}$ Manifestations of intestinal malrotation are uncommon in adulthood. Symptomatic patients may present with either acute abdominal pain due to entire or segmental midgut volvulus, or chronic abdominal pain due to proximal midgut partial obstruction in the presence of abnormal congenital bands. A limited number of cases of duodenal obstruction associated with midgut malrotation has been reported in the medical literature. ${ }^{(2-5)}$ We herein report an unusual case of duodenal obstruction associated with midgut malrotation due to organoaxial partial rotation of the distal duodenum. This is probably the first of such a case diagnosed in an adult reported in the medical literature.

\section{CASE REPORT}

A 57-year-old Sri Lankan woman complained of abdominal bloating, episodes of vomiting of bilious contents and epigastric pain following main meals for the last two years. These episodes were initially infrequent and occurred only two to three times a week. As the frequency of such episodes increased, she decided to seek medical advice. She had never experienced dyspeptic symptoms or upper gastrointestinal bleeding manifestations previously. She had neither a history of ingesting corrosive agents nor a family history of upper gastrointestinal malignancies. She had also never undergone any abdominal surgical procedures. She had lost about $10 \mathrm{~kg}$ within two months. She had been admitted twice during the two-month period to another hospital, where she received conservative management prior to the current admission. She had undergone oesophagogastroduodenoscopy and ultrasonography of the abdomen, with unremarkable results.
She was moderately pale on examination and her body weight was $49 \mathrm{~kg}$, with a body mass index of $21.1 \mathrm{~kg} / \mathrm{m}^{2}$. She was anicteric and there was no lymphadenopathy. Abdominal examination did not reveal any mass lesion, organomegaly or ascitis. Succussion splash was not elicited.

Her haemoglobin $(9.2 \mathrm{~g} / \mathrm{dL})$ and serum albumin levels $(27 \mathrm{~g} / \mathrm{L})$ were low. Liver transaminases and renal function tests were unremarkable. Radiological imaging favoured a diagnosis of duodenal obstruction. Barium meal and follow through showed grossly dilated second and third parts of the duodenum, with an abrupt cutoff at the third part (Fig. 1). Postural changes during the barium meal procedure resulted in some degree of delayed emptying of barium beyond the assumed area of obstruction. The stomach and oesophagus were normal. Findings of the barium meal suggested the possibility of Wilkie's syndrome or compression of the ligament of Treitz. Contrast-enhanced computed tomography (CT) confirmed the dilation of the proximal duodenum and revealed evidence of midgut malrotation with the superior mesenteric artery lying posterior to the duodenum, excluding Wilkie's syndrome (Fig. 2). Therefore, we entertained the working diagnosis of duodenal partial obstruction associated with midgut malrotation.

Following detailed discussions with the patient about the current problem, a bypass surgical procedure was planned as the best available management option. She underwent an exploratory midline laparotomy in October 2012. Findings of the peritoneal survey included evidence of midgut malrotation with the caecum lying on the right subhepatic space and small bowel loops occupying the right side of the abdomen. The duodenum was situated beneath the caecum and proximal ascending colon. Slender congenital bands related to the large bowel were present without compression of the underlying duodenum. Limited mobilisation of the caecum exposed the 


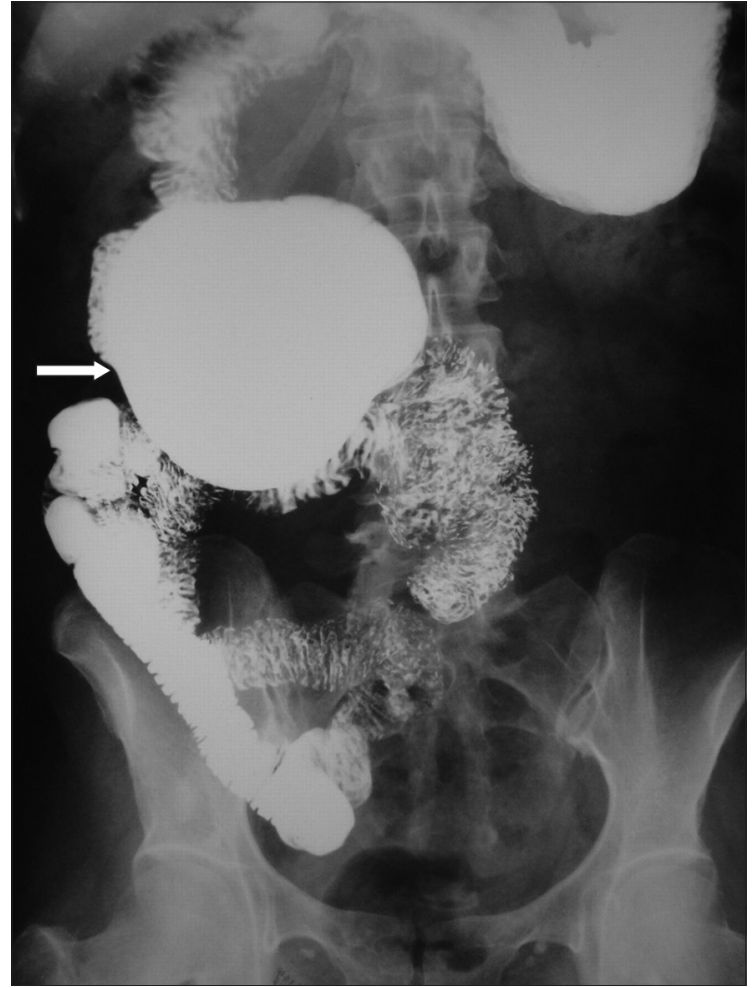

Fig. 1 Delayed film of the barium meal shows the dilated segment of the proximal duodenum retaining the contrast (arrow), and the abnorma position of the small bowel entirely occupying the right side of the abdomen.

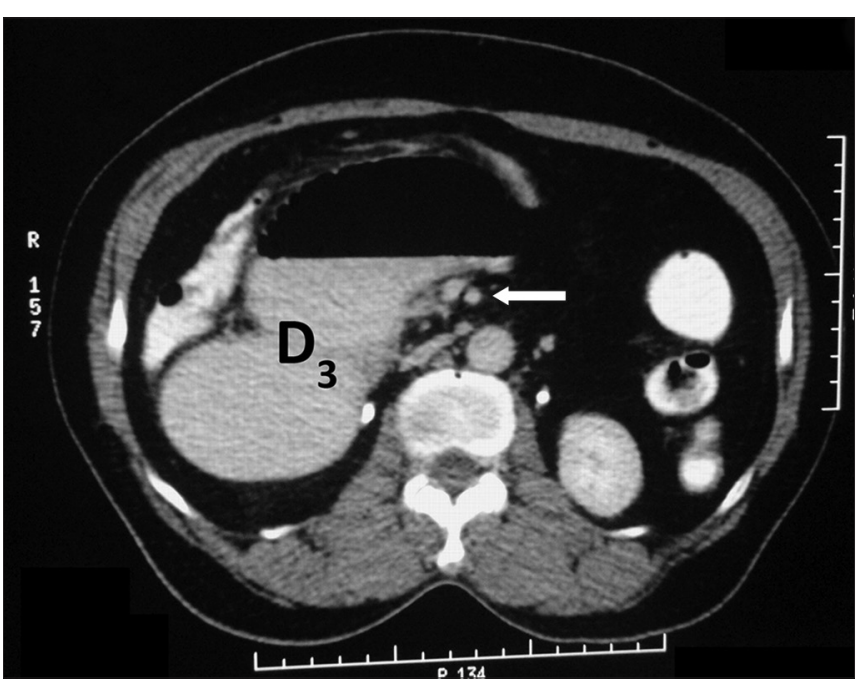

Fig. 2 Horizontal section of the contrast enhanced CT at the level of the duodenum shows the dilated third part of the duodenum $\left(D_{3}\right)$, and the abnormal position of the superior mesenteric artery lying posterior to the duodenum (arrow).

distended and hypertrophied proximal duodenum up to its third part. The third part of the duodenum contained a segment of organoaxial partial twisting, which has led to proximal obstruction (Figs. 3a \& b). The superior mesenteric artery was not visualised over the distal segment of the duodenum. Full mobilisation of the duodenum was not attempted due to the deranged anatomy. A Roux-en-Y duodenojejunostomy was performed using a single layer $3 / 0$ polyglactin suture anastomosis. As the mesenteric axis was wide, the Ladd procedure was not performed. The patient's postoperative period was uncomplicated. Oral feeding was commenced on postoperative Day 3 and the patient tolerated solid meals by Day 7 . At the time of the latest review (nine months since the surgical procedure), the patient was completely free of previous symptoms and had gained $15 \mathrm{~kg}$.

\section{DISCUSSION}

The midgut undergoes a complex process of rotation outside the fetal abdomen during embryological development. Midgut malrotation is a spectrum of congenital anomalies referring to the absence, lack or incomplete rotation of the fetal intestine around the axis of the superior mesenteric artery. ${ }^{(6)}$ This results in various abnormalities in the positioning of intestinal organs, such as the small bowel predominantly occupying the right side of the abdomen, the caecum displaced to the epigastrium or right hypochondrium, and anomalies of the ligament of Treitz with inferior and rightward displacement. These malpositions of the small intestine lead to an unusually narrow base at the root of the mesentery. In the presence of such anomalies, the midgut is prone to volvulus, causing intestinal ischaemia. ${ }^{(7)}$ Additionally, thick fibrous bands of Ladd over the duodenum may cause compression. ${ }^{(7,8)}$ The exact incidence of intestinal rotation disorders is unknown. Autopsy studies have estimated that it may be as high as $1 \%$ of the total population. ${ }^{(9)}$ Gut malrotation is generally regarded as a paediatric pathology. It is believed that about $50 \%$ of cases would present during the first week of life and about $80 \%$ may present within the first month of life. ${ }^{(1)}$ Manifestations of gut malrotation are rare in the adult population. Symptomatic patients may present with either acute small bowel obstruction and intestinal ischaemia associated with midgut volvulus, or chronic vague intermittent abdominal pain due to subacute obstruction.

Intestinal malrotation may be associated with obstruction of the third part of the duodenum mainly due to external compression by the abnormal fibrous bands of Ladd. ${ }^{(8)}$ In addition, there are rare occurrences of intrinsic duodenal obstruction due to duodenal malrotation. Lewis reported the occurrence of partial duodenal obstruction with incomplete duodenal rotation. ${ }^{(10)}$ Firor and Harris documented the coexistence of isolated incomplete duodenal rotation in the presence of complete rotation of the colon, and demonstrated the potential of such abnormalities to produce partial duodenal obstruction by faulty mesenteric fixation, where the duodenum is angulated or kinked. ${ }^{(11)}$ They also highlighted the abnormal positioning of the duodenum in relation to the superior mesenteric artery and commented on the possibility of intrinsic duodenal obstruction. Ablow et al presented the management experience of four cases of upper gastrointestinal obstruction due to a Z-shaped duodenojejunal loop, which they attributed as a sign of mesenteric fixation anomaly. ${ }^{(12)}$ All these patients were diagnosed within the paediatric age group. The deranged duodenal anatomy identified in our patient is compatible with the description given by Firor and Harris, and this is probably the first of such a case diagnosed in an adult.

The upper gastrointestinal contrast studies are considered as the standard basic imaging modality to detect midgut malrotation. ${ }^{(11)} \mathrm{CT}$ is useful for the accurate diagnosis of intestinal 

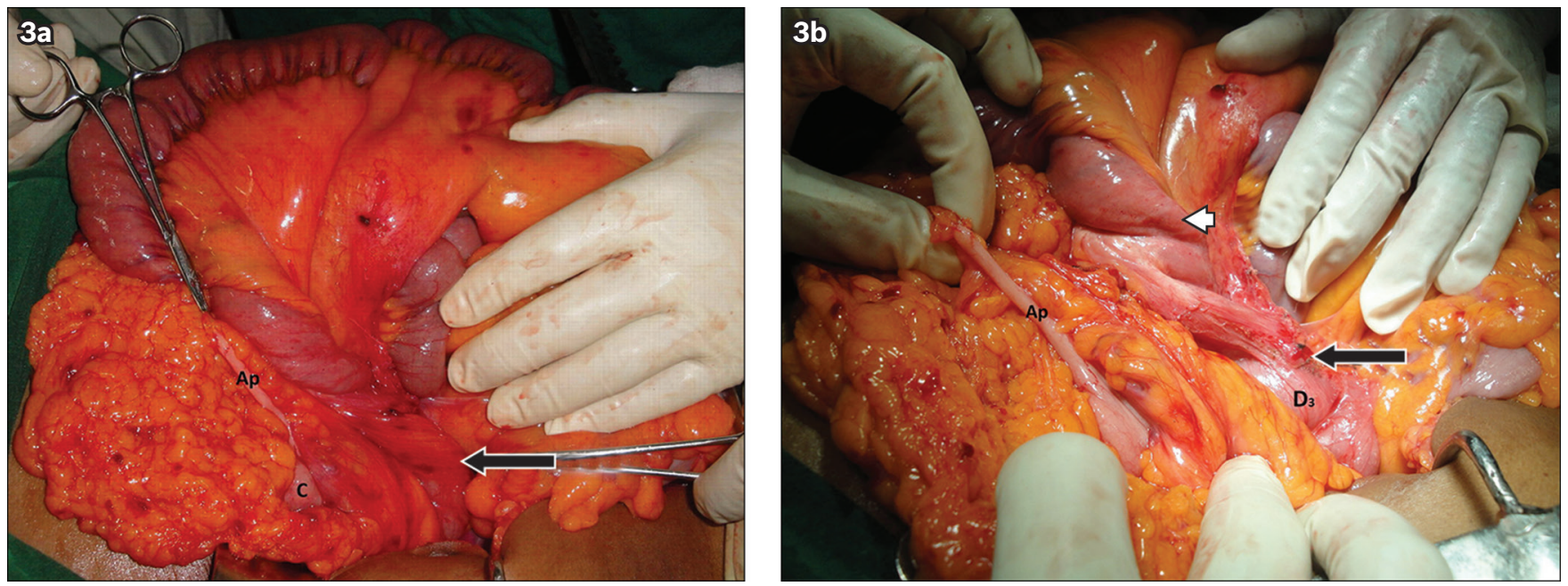

Fig. 3 (a) Perioperative photograph shows the duodenal obstruction due to axial rotation (arrow), and the subhepatic position of the caecum (C) and appendix (Ap). (b) Close-up photograph of the duodenal obstruction, including the appendix (Ap) and dilated third part of the duodenum ( $\mathrm{D}_{3}$ ), shows the point of organoaxial rotation of the duodenum (arrow) and the duodenojejunal junction (arrowhead).

malrotation, as it shows not only the malpositioned intestine but also other associated extraintestinal anomalies such as the malposition of the superior mesenteric vasculature. ${ }^{(13)}$ In our case, barium meal and follow through helped to identify the partial duodenal obstruction and midgut malrotation. CT was useful in excluding superior mesenteric artery syndrome during the preoperative preparation. However, the exact cause of the obstruction was only confirmed at exploration. Surgery remains the mainstay of treatment for symptomatic gut malrotation regardless of age, and Ladd procedure is commonly performed to prevent midgut volvulus. In the management of our patient, bypass Roux-en-Y duodenojejunostomy was performed as her symptoms were solely related to the duodenal obstruction. As the mesenteric axis was adequately wide, Ladd procedure was not considered. Success of the surgery was assessed by symptomatic relief and weight gain of the patient within the first six months after surgery.

This rare case highlights the possibility of congenital duodenal obstruction presenting in adulthood. It provides the valuable opportunity for surgeons to be aware of such occurrences, which need to be considered as a cause for adult duodenal obstruction other than for neoplastic diseases and extraluminal compressions.

\section{ACKNOWLEDGEMENTS}

We acknowledge the valued contribution of Dr Dishna de Silva, Consultant Paediatrician, Lady Ridgeway Hospital,
Colombo, Sri Lanka, for her advice in the preparation of this article.

\section{REFERENCES}

1. von Flüe M, Herzog U, Ackermann C, Tondelli P, Harder F. Acute and chronic presentation of intestinal non rotation in adult. Dis Colon Rectum 1994; 37:192-8.

2. Gong J, Zheng ZJ, Mai G, Liu XB. Malrotation causing duodenal chronic obstruction in an adult. World J Gastroenterol 2009; 15:1144-6.

3. Basu S, Srivastava V, Singh PK, Srivastava A, Shukla VK. Duodenojejunal junction web masquerading as Wilkie's syndrome: report of a case. Surg Today 2011; 41:409-11.

4. Hinkle JK, Smith TR. Malrotation with volvulus of the transverse colon and duodenal obstruction secondary to Ladd's bands. Clin Imaging 2008; 32:65-8.

5. Chen CC, Wang SS, Hwang JI, et al. Midgut malrotation with partial obstruction of duodenojejunal junction due to Ladd's bands. J Clin Gastroenterol 1991; 13:590-3.

6. Zissin R, Rathaus $\mathrm{V}$, Oscadchy $\mathrm{A}$, et al. Intestinal malrotation as an incidental finding on CT in adults. Abdom Imaging 1999; 24:550-5.

7. Fu T, Tong WD, He YJ, et al. Surgical management of intestinal malrotation in adults. World J Surg 2007; 31:1797-803.

8. Ladd WE. Surgical diseases of the alimentary tract in infants. N Engl J Med 1936; 215:705-8.

9. Kapfer SA, Rappold JF. Intestinal malrotation-not just the pediatric surgeon's problem. J Am Coll Surg 2004; 199:628-35.

10. Lewis JE Jr. Partial duodenal obstruction with incomplete duodenal rotation. J Pediat Surg 1966; 1:47-53.

11. Firor HV, Harris VJ. Rotational abnormalities of gut. Re-emphasis of neglected facet, isolated incomplete rotation of duodenum. Am J Roentgenol Radium Ther Nucl Med 1974; 120:315-21.

12. Ablow RC, Hoffer FA, Seashore JH, Touloukian RJ. Z-shaped duodenojejunal loop: sign of mesenteric fixation anomaly and congenital bands. AJR Am J Roentgenol 1983; 141:461-4.

13. Fisher JK. Computer tomographic diagnosis of volvulus in intestinal malrotation. Radiology 1981; 140:145-6. 